\section{Simultaneous in vivo dynamic magnetic resonance-diffuse optical tomography for small animal imaging}

\author{
Mehmet Burcin Unlu, ${ }^{*}$ Yuting Lin, Ozlem Birgul, \\ Orhan Nalcioglu, and Gultekin Gulsen \\ University of California, Tu and Yuen Center for Functional \\ Onco-Imaging, Irvine, California 92697 \\ E-mail:munlu@uci.edu
}

\begin{abstract}
We present simultaneous measurement of enhancement kinetics of an optical and a magnetic resonance (MR) contrast agent in a small animal breast tumor model (R3230 ac) using a combined MR-diffuse optical tomographic (MR-DOT) imaging system. A mixture of a small molecular-weight MR contrast agent gadoliniumdiethylene-triamine-pentaacetic acid (Gd-DTPA) and a large molecular-weight optical contrast agent indocyanine green (ICG) was administered intravenously for multimodal dynamic imaging. Coregistration of optical and MR images was accomplished using agar-water-based markers. Using $T_{2}$ and dynamic $T_{1}$ weighted MR images, we divided the entire tumor into two regions of interest (ROI): a viable and a nonviable region. The absorption enhancements in the ROls were calculated. An enhancement of the ICG was observed in the viable region. On the contrary, there was a lower enhancement in the nonviable region. $\odot 2008$ Society of Photo-Optical Instrumentation Engineers. [DOI: 10.1117/1.3041165]
\end{abstract}

Keywords: medical imaging; tomography; infrared imaging; magnetic resonance imaging; image reconstruction.

Paper 08150LR received May 7, 2008; revised manuscript received Oct. 1, 2008; accepted for publication Oct. 2, 2008; published online Dec. 18, 2008. This paper is a revision of a paper presented at the SPIE conference on Multimodal Biomedical Imaging II, January 2007, San Jose, California. The paper presented there appears (unrefereed) in SPIE Proceedings Vol. 6431.

\section{Introduction}

Diffuse optical tomography (DOT) can be used to localize and characterize a tumor based on endogenous contrast. Besides, using exogenous contrast agents may provide additional information for tumor detection and characterization. For example, it may be possible to use the dynamic behavior of indocyanine green (ICG) for differential diagnosis. ${ }^{1-3}$ ICG binds to plasma proteins; thus, it is confined to the vascular compartment and behaves as an intravascular contrast agent.

To date, contrast agents have been used extensively in the magnetic resonance (MR) imaging of tumors including breast cancer. ${ }^{4}$ Clinical studies have shown that dynamic contrast enhanced-MR imaging (DCE-MRI) has a high sensitivity in breast cancer detection. Nonetheless, the major obstacle on

*Tel.: 9498245012; Fax: (949) 824-3481; E-mail: munlu@uci.edu the road to the use of DCE-MRI in breast cancer diagnosis is its low specificity. This is due to the small molecular weight of gadolinium-diethylene-triamine-pentaacetic acid (Gd-DTPA, $0.57 \mathrm{kDa}$ ) that leaks out of tumor vasculature. ${ }^{5}$ As a result, some benign lesions may show malignant-type enhancement kinetics and give false positive results because of their high vascularity and high interstitial volume.

This study is motivated by the desire to use a mixture of a small and a large molecular agent to improve specificity in cancer imaging. ${ }^{5}$ Small molecular agents leak into the extravascular space in tumors, resulting in higher contrast that improves sensitivity. Meanwhile, the large molecular optical agent probes vascular permeability, resulting in improved specificity. In this letter, we report the simultaneous measurement of optical and MR contrast agent kinetics using a frequency domain DOT small animal imaging system with a time resolution of $16 \mathrm{~s}$ and a $4 \mathrm{~T}$ MRI system.

\section{Methods}

We used a R3230 ac-induced small animal breast tumor model in this study. Tumor cells were injected into a Fisher rat that weighed $170 \mathrm{~g}$. The tumor size was $\sim 2 \mathrm{~cm}$. We used a frequency domain DOT system that had already been integrated into a 4T MR scanner previously. ${ }^{6}$ The time resolution of the imaging system was a pivotal feature of the dynamic studies. Our frequency domain DOT system has eight sources and eight detectors ${ }^{7}$ with a time resolution of $16 \mathrm{~s}$ per time point. The time resolution of dynamic MR data acquisition was set to $23 \mathrm{~s}$. Optical measurements were performed with a laser diode emitting at $785 \mathrm{~nm}$ and modulated at a frequency of $100 \mathrm{MHz}$. The MR and the DOT measurements were started and performed simultaneously. A mixture of Gd-DTPA $(0.1 \mathrm{mmol} / \mathrm{kg})$ and ICG $(0.5 \mathrm{mg} / \mathrm{kg})$ was injected intravenously into the animal after the first three $T_{1}$ weighted baseline acquisitions. Data were acquired by both systems for $\sim 10 \mathrm{~min}$.

Afterward, the calibrated optical data were analyzed using the diffusion equation with the Robin boundary conditions. We used the finite element method for the numerical solution of the diffusion equation. The details of the reconstruction scheme were described in Refs. 6 and 8. In the optical reconstructions, the first time point was selected as the baseline and then it was subtracted from subsequent dynamic optical data. Coregistration of MR and optical images was accomplished using agar-water-based markers attached to DOT fiber probes. ${ }^{9}$ These markers were visible on the MR images so that contact points of the fibers on the animal surface could be located. Please note that, in this preliminary study, we did not use any anatomical a priori information in the optical image reconstruction so as not to bias the inverse solution. It was important to take into account the heterogeneity of the tumor when calculating the contrast agent kinetics. The availability of both the $T_{1}$ and $T_{2}$-weighted images made it possible to select a viable and a nonviable region in the tumor. The two main criteria that characterized a viable region were $(i)$ high signal (bright regions) on the MR enhancement image and (ii) normal or low signal (dark regions) in the $T_{2}$ image. ${ }^{9}$ Accordingly, a nonviable region of the tumor was defined as a region that did not show $T_{1}$ signal enhancement. We divided the

1083-3668/2008/13(6)/060501/3/\$25.00 @ 2008 SPIE 


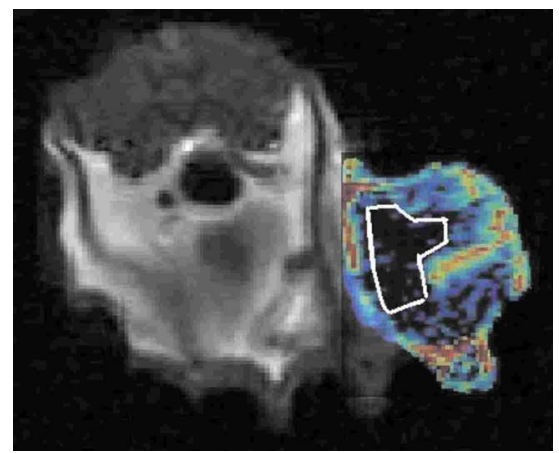

(a)

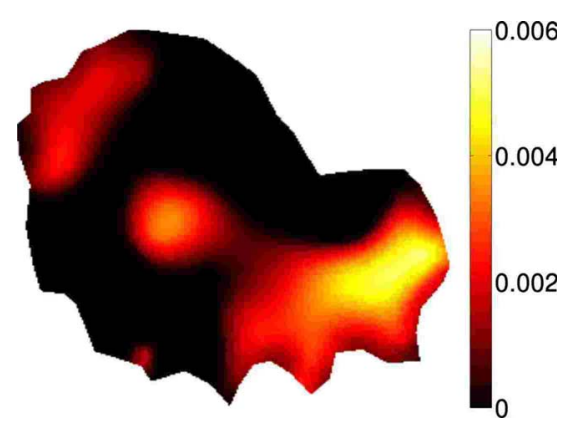

(b)

Fig. 1 Enhancement images are given. (a) $T_{1}$-weighted MR image at the peak signal enhancement. Red corresponds to the highest enhancement spots. The ROI covering the nonviable part of the tumor is shown on the image. (b) Reconstructed $\delta \mu_{\mathrm{a}}$ map, at $t=160 \mathrm{~s}$. (Color online only.)

entire tumor into two regions: one viable and one nonviable region. These ROIs were selected from MR enhancement images corresponding to the optical imaging slice. After the DOT reconstruction was completed, the mean of reconstructed absorption in each ROI at each time point was used to obtain the fractional absorption enhancement curve that was defined as $\left[\mu_{a}(t)-\mu_{a}(t=0)\right] / \mu_{a} \quad(t=0)$. Similarly, fractional MR signal enhancement was defined $\left(S_{\mathrm{T}_{1}(t)}-S_{\mathrm{T}_{1}(t=0)}\right) / S_{\mathrm{T}_{1}(t=0)}$.

\section{Results}

Figure 1(a) displays the enhancement image superimposed on the $T_{1}$-weighted image of the rat cross section. The ROI that was displayed on the image covered the nonviable region. The rest of the tumor was considered to be viable. Figure 1(b) shows the reconstructed absorption map at $t=160 \mathrm{~s}$, when maximum enhancement was obtained. The calculated mean $\mu_{a}$ was $0.019 \mathrm{~mm}^{-1}$ in the viable ROI for the baseline measurement, and it increased to $0.022 \mathrm{~mm}^{-1}$ at the peak time point. Meanwhile, mean $\mu_{a}$ increased to $0.019 \mathrm{~mm}^{-1}$ in the nonviable ROI.

Figure 2(a) shows the fractional enhancement for the $T_{1}$-weighted signal obtained by MRI from the nonviable and viable ROIs. It was clear that the nonviable region exhibited low enhancement, and there was a higher signal enhancement in the viable region. In the viable ROI, Gd-DTPA kinetics showed a rapid rise. This was because small molecular contrast agent leaked out of the blood vessels in the viable region

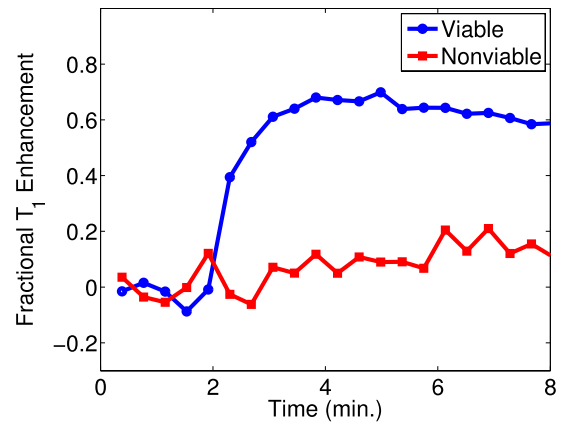

(a)

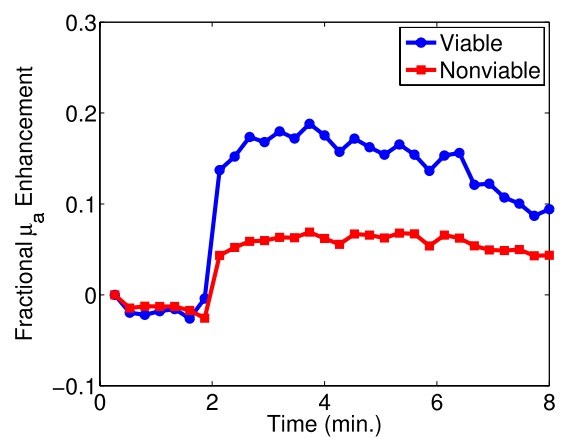

(b)

Fig. 2 (a) Fractional $T_{1}$ enhancement and (b) fractional $\mu_{a}$ enhancement in the viable and nonviable region.

causing a rapid increase in the signal that reached the peak point immediately after the injection. The slow decay seen afterward showed that the Gd-DTPA started diffusing back into the intravascular space.

Optical absorption enhancement kinetics is displayed in Fig. 2(b). Absorption kinetics showed a rapid rise in the viable region similar to MRI, and a slow decay was observed. The large molecular size of albumin-ICG made it difficult for the contrast agent to exchange between the extravascular and intravascular spaces. Meanwhile, the kinetics in the nonviable ROI showed low enhancement.

\section{Discussion}

DOT imaging allowed spatially resolved measurement of absorption kinetics within the tumor. The 16-s temporal resolution of our system allowed us to measure the fast-rising slope of absorption enhancement and recover the peak value of the optical absorption. It had been shown that reduced scattering did not depend on the concentration of ICG significantly. ${ }^{10}$ Therefore, we calculated only the absorption kinetics.

The spatial resolution of DOT was low compared to the MRI. As a result, the recovered optical enhancement map did not entirely overlap with the MR enhancement map. This showed that structural a priori information obtained from MRI should be used in optical reconstruction.

Some qualitative features of both contrast agents could be observed from the kinetic curves in the viable ROI. The kinetics of Gd-DTPA displayed the characteristics of a small molecular weight agent, whereas ICG kinetics showed the features of a large molecular agent. In addition, neither agent 


\section{JBO LETTERS}

showed a significant enhancement in the nonviable region. These results were consistent with the DCE-MRI literature. ${ }^{11}$

Using two contrast agents, one small molecular weight MR and one large molecular weight optical agent, during a single imaging session provides fully coregistered complementary information. This study was the first step toward the goal to use optical imaging in conjunction with the DCE-MRI to improve specificity in tumor characterization.

\section{Acknowledgments}

We thank Dr. Min-Ying Su and Dr. Hon J. Yu for discussions on the subject. This research was supported in part by the National Cancer Institute through Grants No. R21/33 CA101139, No. R21/33 CA120175, No. R21 CA 121568, and No. U54 CA105480; the California Breast Cancer Research Program No. 12IB-0095; and U.S. Army BC051304.

\section{References}

1. V. Ntziachristos, A. G. Yodh, M. Schnall, and B. Chance, "Concurrent MRI and diffuse optical tomography of breast after indocyanine green enhancement," Proc. Natl. Acad. Sci. U.S.A. 97, 2767-2772 (2000).

2. X. Intes, J. Ripoll, Y. Chen, S. Nioka, A. G. Yodh, and B. Chance, "In vivo continuous-wave optical breast imaging enhanced with indocyanine green," Med. Phys. 30, 1039-1047 (2003).

3. B. Alacam, B. Yazici, X. Intes, S. Nioka, and B. Chance, "Pharmacokinetic-rate images of indocyanine green for breast tumors using near-infrared optical methods," Phys. Med. Biol. 53, 837-859 (2008).

4. E. Warner, D. B. Plewes, R. S. Shumak, G. C. Catzavelos, L. S. D. Prospero, M. J. Yaffe, V. Goel, E. Ram, P. L. Chart, D. E. Cole, G. A. Taylor, M. Cutrara, T. H. Samuels, J. P. Murphy, J. M. Murphy, and S. A. Narod, "Comparison of breast magnetic resonance imaging, mammography, and ultrasound for surveillance of women at high risk for hereditary breast cancer," J. Clin. Oncol. 19, 3524-3531 (2001).

5. M. Y. Su, A. Muhler, X. Lao, and O. Nalcioglu, "Tumor characterization with dynamic-contrast enhanced MRI using MR contrast agents of various molecular weights magnetic resonance in medicine," Magn. Reson. Med. 39, 259-269 (1998).

6. G. Gulsen, M. B. Unlu, O. Birgul, O. Nalcioglu, and F. S. Azar (Eds.), "Simultaneous monitoring of multiple contrast agents using a hybrid MR-DOT system," Proc. SPIE 6431, 64310D (2007).

7. G. Gulsen, B. Xiong, O. Birgul, and O. Nalcioglu, "Design and implementation of a multifrequency near-infrared diffuse optical tomography system," J. Biomed. Opt. 11, 014020 (2006).

8. M. B. Unlu, O. Birgul, R. Shafiiha, G. Gulsen, and O. Nalcioglu, "Diffuse optical tomographic reconstruction using multifrequency data," J. Biomed. Opt. 11, 054008 (2006).

9. S. Merritt, F. Bevilacqua, A. J. Durkin, D. J. Cuccia, R. Lanning, B. J. Tromberg, G. Gulsen, H. Yu, J. Wang, and O. Nalcioglu, "Coregistration of diffuse optical spectroscopy and magnetic resonance imaging in a rat tumor model," Appl. Opt. 42, 2951-2959 (2003).

10. B. Yuan, N. Chen, and Q. Zhu, "Emission and absorption properties of indocyanine green in Intralipid solution," J. Biomed. Opt. 9, 497503 (2004)

11. M. Y. Su, Z. Wang, P. M. Carpenter, X. Lao, A. Muhler, and O. Nalcioglu, "Characterization of N-ethyl-N-nitrosourea-induced malignant and benign breast tumors in rats by using three MR contrast agents," J. Magn. Reson Imaging 9, 177-186 (1999). 